\title{
Modeling the Hysteresis Loop of the Nanocomposite Material Using Modified Hyperbolic $\boldsymbol{T}(\boldsymbol{x})$ Model
}

\author{
M. DośPiAe* \\ Częstochowa University of Technology, Faculty of Production Engineering and Materials Technology, \\ Institute of Physics, al. Armii Krajowej 19, 42-200 Częstochowa, Poland
}

\begin{abstract}
The paper presents possibilities of hysteresis loop decomposition onto the magnetization components. The reversible and irreversible magnetization changes describe processes, which are influencing reversal magnetization in studied permanent magnets. Further, these components are used for modelling the recoil curves using modified hyperbolic $T(x)$ model.
\end{abstract}

DOI: $10.12693 /$ APhysPolA.127.415

PACS: $75.40 . \mathrm{Mg}, 75.60 . \mathrm{Jk}, 75.60 . \mathrm{Jk}, 75.30 . \mathrm{Et}, 75.50 \mathrm{Ww}$

\section{Introduction}

The phenomenon of magnetic hysteresis is inextricably linked with permanent magnets. Since many years, it is well known that in order to have good functional properties, the permanent magnet must have shape of the central part of the hysteresis loop similar, as much as possible to the rectangle, and must be characterized by a high value of saturation of polarization. In fact, its shape in the center of the coordinate system deviates from the rectangle and strongly depends on sample phase composition, structure, and defects occurring in it. In turn, these factors are directly shaping the reversal magnetization mechanisms and interactions that occur between grains in magnetic material [1-4].

For example, single-phase magnets are characterized by smooth course of the magnetic hysteresis loop, irrespective of the grain size. If the grain size in such magnet is lower than the exchange interactions length, the remanence is greater than half of the saturation magnetization and the ratio $M_{\mathrm{R}} / M_{S}>0.5$. The increase in grain size will not cause constriction in the central part of the hysteresis loop, will only result in a change of the $M_{\mathrm{R}} / M_{\mathrm{S}}$ ratio (change in slope of the loop). The constriction, in the central part of the hysteresis loop, will occur in case of grain growth in multiphase magnets, due to loss of exchange coupling between the magnetic moments of phases with different magnetic hardness [5-7].

The effect of aforementioned factors (structure, phase composition i.e.) on the reversible and irreversible magnetization changes and the shape of hysteresis loop is more complex and usually determined on the basis of recoil curves compiled with results of structure analysis [2$5]$. These relations are not the point of this paper and will not be discussed in detail here.

The aim of this paper was to model magnetic hysteresis loop taking into account reversal magnetization mecha-

* corresponding author; e-mail: mdospial@wp.pl nisms, occurring in magnetic nanocomposites. Then, applying the parameters used in modeling hysteresis loop for simulation of recoil curves.

\section{Hysteresis model}

According to Takacs [8], hysteresis loop can be described by the combination of sigmoid and linear functions, characterizing properly irreversible and reversible magnetization changes. The same model was adapted by the Author in order to model hysteresis loop of hard magnetic material [9]. In this paper Author is proposing modification of the $T(x)$ model involving description of reversible magnetization component by anhysteretic function. Basing on this assumption the whole reversal magnetization process occurring in studied hard magnetic material have been described by the following mathematical equations:

$$
\begin{aligned}
& f_{r,+}^{\text {hys }}=\mu_{0} M_{\mathrm{R}} \sum_{i=1}^{n_{\mathrm{irr}}} B_{0, i}\left(\tanh \left(C_{0, i}\left(x-a_{0, i}\right)\right)+b_{+, i}-b_{1, i}\right),( \\
& f_{r,+}^{\text {anhys }}=\mu_{0} M_{\mathrm{rev}}^{\max } \\
& \times \sum_{j=1}^{n_{\mathrm{rev}}} B_{0, j} \frac{\tanh \left(C_{0, j}\left(x+a_{0, j}\right)\right)+\tanh \left(C_{0, j}\left(x-a_{0, j}\right)\right)}{2},(1 \mathrm{~b}) \\
& b_{+, i}=\tanh \left(C_{0, i}\left(x_{k}+a_{0, i}\right)\right)-\tanh \left(C_{0, i}\left(x_{k}-a_{0, i}\right)\right), \\
& f_{r,-}^{\text {hys }}=\mu_{0} M_{\mathrm{R}} \sum_{i=1}^{n_{\mathrm{rrr}}} B_{0, i}\left(\tanh \left(C_{0, i}\left(x+a_{0, i}\right)\right)+b_{-, i}+b_{1, i}\right), \\
& f_{r,-}^{\text {anhys }}=\mu_{0} M_{\mathrm{rev}}^{\max } \\
& \times \sum_{j=1}^{n_{\mathrm{rev}}} B_{0, j} \frac{\tanh \left(C_{0, j}\left(x+a_{0, j}\right)\right)+\tanh \left(C_{0, j}\left(x-a_{0, j}\right)\right)}{2}, \\
& b_{-, i}=\tanh \left(C_{0, i}\left(x_{k}-a_{0, i}\right)\right)-\tanh \left(C_{0, i}\left(x_{k}+a_{0, i}\right)\right) \\
& b_{1, i}=\frac{\tanh \left(C_{0, i}\left(x_{m}+a_{0, i}\right)\right)-\tanh \left(C_{0, i}\left(x_{m}-a_{0, i}\right)\right)}{2}
\end{aligned}
$$

where $f_{ \pm}^{\text {hys,anhys }}$ are ascending $(+)$ and descending (-) functions, representing reversible (anhys) and irreversible (hys) magnetization components, respectively, 
$x$ is the field excitation, $a_{0 i}$ is the center of $i$-th pinning/nucleation site, $a_{0 i}$ is the center of $j$-th reversible process, $B_{0, i}, B_{0, j}$ are the amplitudes of the $i$-th and $j$ th magnetization components, $C_{0, i}, C_{0, j}$ are the sheering factors, while $x_{m}$ represents the maximum field excitation. The $i$ and $j$ indexes are referring to the individual reversible and irreversible magnetization component, respectively and $n_{\text {irr,rev }}$ is their total number.

\section{Experimental procedure}

The studied sample of $\mathrm{Sm}_{10.53} \mathrm{Co}_{57.89} \mathrm{Fe}_{22.79} \mathrm{Zr}_{1.74}$ $\mathrm{Cu}_{7.05}$ alloy was manufactured by HDDR technique. Comprehensive structure studies were published elsewhere [10]. Obtained alloy had large grains, of $1 \mu \mathrm{m}$ diameter and cluster-type of structure. Clusters consisted of alternating $\mathrm{SmCo}_{5}$ and $\mathrm{Sm}_{2} \mathrm{Co}_{17}$ phases, of nanometric sizes. Therefore, the studied material was treated as a nanocomposite.

The measurements of static hysteresis loop and recoil curve were done using LakeShore VSM with maximum external magnetic field of $2 \mathrm{~T}$. The method of recoil curves decomposition of the magnetization components was described elsewhere [11]. The sample used for magnetic measurements had a cylinder shape of known dimensions i.e. diameter $0.3 \mathrm{~cm}$ and height of $1 \mathrm{~cm}$. The demagnetization field resulting from its shape was taken into account and evaluated by the method described in [12].

\section{Results and discussion}

The magnetic measurements of recoil curves have been used to determine the reversible and irreversible magnetization components and their susceptibilities (Fig. 1). Basing on obtained results, the magnetization processes occurring in studied material were identified and three characteristic ranges, resulting from different types of processes have been marked in Fig. 1b. The range (I) represents reversible magnetization changes resulting from rotation of magnetization vector. Next, range (II) in literature is attributed to magnetization changes resulting from the motion of domain walls after their unpinning in multidomain grains or bowing of strongly pinned domain walls [13-15]. Finally, the range (III) represents irreversible magnetization changes resulting from unpinning of domain walls $[4,16]$.

To each of marked ranges has been assigned sech distribution function. Basing on proper magnetic susceptibility components, the parameters describing aforementioned processes have been determined and shown in Table. Designated parameters have been used to simulate the hysteresis loop. In Fig. 2 there are presented hysteretic and anhysteretic functions representing irreversible and reversible processes occurring in studied sample, during reversal magnetization, respectively. Basing on these results, the sum of both function representing total magnetization changes has been compared with experimental demagnetization curve. It can be stated that simulated hysteresis loop shows high compliance with the experimental data.

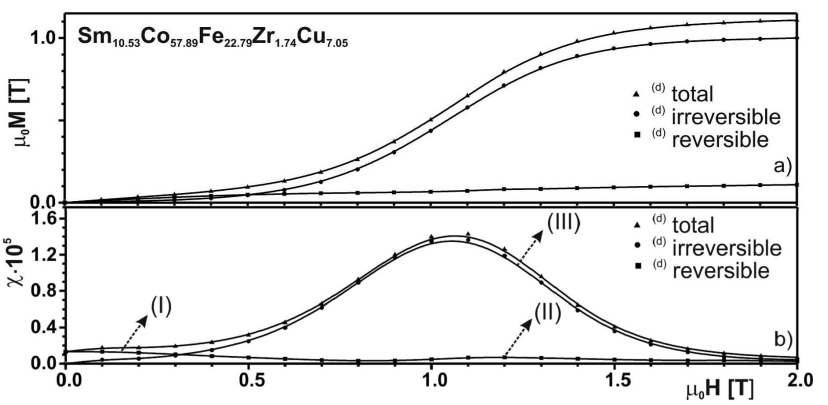

Fig. 1. Total, reversible and irreversible magnetization changes (a) and corresponding to them magnetic susceptibilities (b).

TABLE

Fitting parameters of distribution functions determined from decomposition of susceptibility components.

\begin{tabular}{|c|c|c|c|c|}
\hline Component & ber & $C_{0 i}$ & $a_{0 i}$ & $B_{0 i}$ \\
\hline \multicolumn{5}{|c|}{$\mathrm{Sm}_{10.53} \mathrm{Co}_{57.89} \mathrm{Fe}_{22.79} \mathrm{Zr}_{1.74} \mathrm{Cu}_{7.05}$} \\
\hline \multirow{2}{*}{ reversible } & 1 & 0.326 & 0.980 & 0.065 \\
\hline & 2 & 3.595 & 0.001 & 0.010 \\
\hline \multirow{2}{*}{ irreversible } & 1 & 4.149 & 1.134 & 0.132 \\
\hline & 2 & 2.754 & 1.027 & 0.868 \\
\hline
\end{tabular}

In original $T(x)$ model reversible magnetization changes are described by linear function so its susceptibility should be constant. The experimental reversible susceptibility dependence has been found to have nonlinear character, similar to distribution function. Basing on obtained results can be stated that reversible magnetization changes should be described by cumulative sigmodal function and should not exhibit hysteresis effect, therefore can be described by anhysteretic function.

The obtained parameters have been also used for simulation of recoil curves basing on Eqs. (1)-(3). The comparison of measured and simulated recoil curves, using modified and original hyperbolic $T(x)$ model, have been presented in Fig. 3. As it can be seen experimental

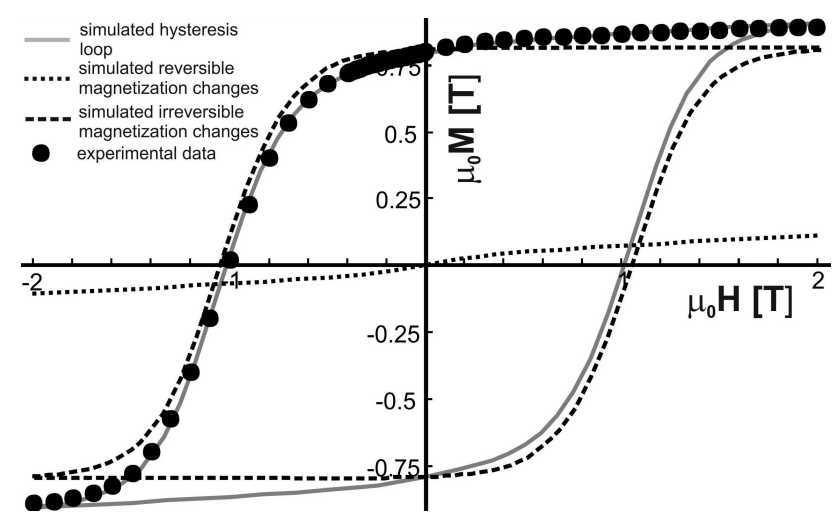

Fig. 2. Measured and simulated demagnetization curves composed from sum of hysteretic and anhysteretic functions representing irreversible and reversible processes. 
and simulated curves practically overlap each other. The presence of small variations between both curves can be explained by time dependent magnetic viscosity. Less compliance, with experimental data, was achieved for functions used in original $T(x)$ model. The differences occurred in both components, due to assignment of some parts of reversible magnetization changes, to the irreversible changes. That confirms the validity of adopted modifications to the $T(x)$ model, consisting on the replacement of the linear function by anhysteretic in description of the reversible magnetization changes.

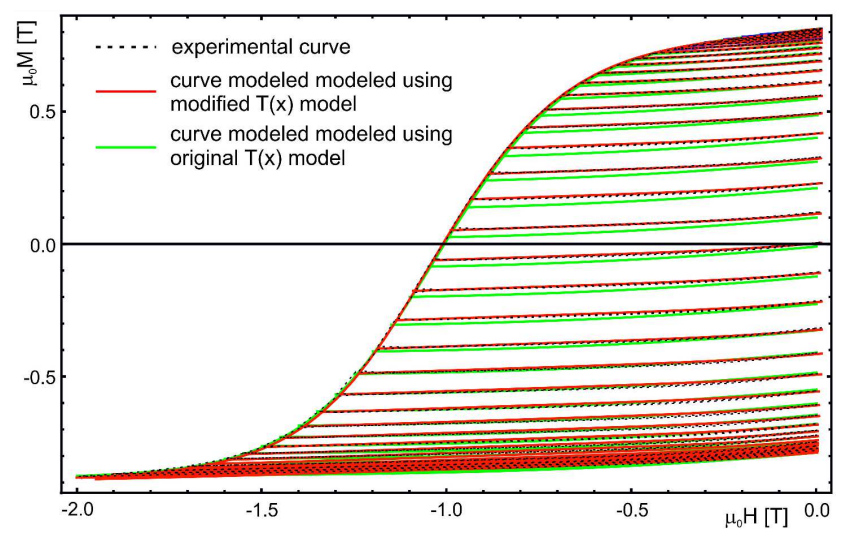

Fig. 3. Measured and simulated, using modified and original hyperbolic $T(x)$ model, recoil curves in demagnetization direction.

\section{Conclusions}

The reversible and irreversible components, are contributing valuable information about the magnetization processes occurring in studied magnets. Basing on reversible component it has been found that for the initial values of applied field, the magnetization changes are mainly resulting from the rotation of magnetization vector. In turn, the reversible changes occurring for the values of external magnetic field close to the coercivity of the sample are attributed to bowing of strongly pinned domain walls or in motion of unpinned domain walls $[2,4,16]$.

Reversible changes do not show a linear relationship, and should be described by anhysteretic function.

The irreversible magnetization changes are resulting from pinning of domain walls on structural defects and grain boundaries $[3,4,16]$.
Analysis of irreversible and reversible components allow to determine the shape of hysteretic and anhysteretic functions contributing to reversal magnetization processes.

\section{Acknowledgments}

This work was supported by the Ministry of Science and Higher Education of Poland: grant no. N N507 234940.

\section{References}

[1] H. Kronmüller, M. Fähnle, Micromagnetism and the Microstructure of Ferromagnetic Solids, Cambridge University Press, United Kingdom 2003.

[2] M. Dospial, M. Nabialek, M. Szota, D. Plusa, J. Alloys Comp. 509S, S404 (2011).

[3] A. Manaf, R.A. Buckley, H.A. Davies, J. Magn. Magn. Mater. 101, 360 (1991).

[4] M. Dospial, D. Plusa, B. Slusarek, J. Magn. Magn Mater. 324, 843 (2012).

[5] J.M.D. Coey, Solid State Commun. 102, 101 (1997).

[6] H. Kronmüller, R. Fischer, M. Bachmann, T. Leineweber, J. Magn. Magn. Mater. 203, 12 (1999).

[7] K. Chu, Z.Q. Jin, V. Chakka, J.P. Liu, J. Phys. D Appl. Phys. 38, 4009 (2005).

[8] J. Takacs, Mathematics of Hysteresis Phenomena, Wiley-VCH Verlag, Weinheim 2003.

[9] M. Dospial, M. Nabialek, M. Szota, P. Pietrusiewicz, K. Gruszka, K. Bloch, Acta Phys. Pol. A 126, 170 (2014).

[10] U. Kotlarczyk, Ph.D. Thesis, Częstochowa University of Technology, Częstochowa 2008 (in Polish).

[11] M. Dospial, D. Plusa, J. Magn. Magn. Mater. 330 , 152 (2013).

[12] H. Zijlstra, in: Ferromagnetic Materials, Ed. E.P. Wohlfarth, North-Holland, Amsterdam 1982, p. 337.

[13] D.C. Crew, P.G. McCormick, R. Street, J. Appl. Phys. 86, 3278 (1999).

[14] D.R. Cornejo, A. Medina-Boudri, H.R. Bertorello, J. Matutes-Aquino, J. Magn. Magn. Mater. 242245, 194 (2002).

[15] M. Dospial, D. Plusa, J. Magn. Magn. Mater. 330, 152 (2013).

[16] M.J. Dospial, M.G. Nabialek, M. Szota, T. Mydlarz, K. Ozga, S. Lesz, J. Alloys Comp. 536S, S324 (2012). 